\title{
A Study on Utilization of Blood Culture Reports in the Emergency Department at a Tertiary Care Teaching Hospital in North India
}

\author{
${ }^{1}$ Sujeet K Sinha, ${ }^{2}$ Anil K Gupta, ${ }^{3}$ Ray Pallab, ${ }^{4}$ Madaan Nirupam
}

\begin{abstract}
Introduction: It is invariably observed that blood cultures are more often ordered because the patient has fever or the admitting physician wants them for any expectation of clinical impact. As a result of such practice, unnecessary blood culture is being ordered with negative financial impact on hospital as well as the patients. The indication for obtaining blood cultures from patients in the Emergency Department (ED) is even less clear. This study was done to study the utilization of blood report in the emergency department of a tertiary care teaching hospital in north India.
\end{abstract}

Need of the study: It was a common perception among the emergency medicine physician that they did not get blood culture reports on time from the Microbiology Department. The reports are either misplaced or received late as desired by the physician. A pilot study was done to check the hypothesis. Totally seventy blood culture samples were sent from the medical emergency ward and surgical emergency ward over a 3 -week period. It was observed that $25 \%$ of total blood culture reports did not reach the physician.

Aim and objective: (1) To study the utilization of blood culture report in emergency, (2) to design an intervention for timely availability of blood culture reports, and (3) to Study the impact of the intervention on the utilization of blood culture reports.

Materials and methods: The study was cross-sectional, record-based prospective study, supplemented with interview of treating doctors. All patients admitted in the emergency ward (one each from medical and surgical) were followed up for 3 months. An Intervention was done personally by the researcher to ensure that all the test reports reach the patient file within 6 hours of its generation.

${ }^{1}$ Consultant, ${ }^{2}$ Medical Superintendent, Professor, and Head ${ }^{3}$ Professor, ${ }^{4}$ Associate Professor

${ }^{1}$ Quality Division, National Health Systems Resource Centre Ministry of Health \& Family Welfare, Government of India, New Delhi, India

${ }^{2}$ Department of Hospital Administration, Postgraduate Institute of Medical Education \& Research, Chandigarh, India

${ }^{3}$ Department of Medical Microbiology, Postgraduate Institute of Medical Education \& Research, Chandigarh, India

${ }^{4}$ Department of Hospital Administration, All India Institute of Medical Sciences, New Delhi, India

Corresponding Author: Sujeet K Sinha, Consultant, Quality Division, National Health Systems Resource Centre, Ministry of Health \& Family Welfare, Government of India, New Delhi India, e-mail: sujeet_sinha20@yahoo.com
Observation: Out of total 104 blood culture reports sent in preintervention, change in treatment according to culture report was done only in $7.6 \%$. In postintervention, it increased to $9.9 \%$. It was found that the clinician did not narrow down treatment even when the cultures are positive. About $77.1 \%$ of positive blood culture reports get wasted in preintervention and $54.5 \%$ in postintervention.

Conclusion: There was ample loss of hospital resources in respect of money as well as manpower if even a single blood culture report is not utilized.

Keywords: Blood culture, Emergency department, Intervention, Utilization.

How to cite this article: Sinha SK, Gupta AK, Pallab R, Nirupam M. A Study on Utilization of Blood Culture Reports in the Emergency Department at a Tertiary Care Teaching Hospital in North India. Int J Res Foundation Hosp Healthc Adm 2018;6(1):12-16.

\section{Source of support: Nil}

Conflict of interest: None

\section{INTRODUCTION}

Bloodstream infections cause significant morbidity and mortality worldwide and are among the most common healthcare-associated infections. Blood culture is a laboratory test to check for bacteria or other microorganisms in a blood sample. It is done when a person has symptoms of a blood infection, also called bacteremia. Blood is drawn from the person one or more times and is tested in a laboratory to find and identify any microorganism present and growing in the blood. If a microorganism is found, more testing is done to determine the antibiotics that will be effective in treating the infection.

Bloodstream infections are now ranked as the 10th leading cause of death in the United States. ${ }^{1,2}$ For this reason, many ED physicians routinely order blood cultures before starting empiric antibiotic therapy in patients they suspect to be bacteremia. However, blood cultures also represent a significant expenditure of resources for hospitals and EDs with no evidence of clinical benefit to admitted patients.

According to the current literature, as many as $90 \%$ of all blood cultures grow no organisms, and of the $10 \%$ that do grow organisms, only about half represent true bacteremia (i.e., true positives), whereas the other half 
are considered contaminants (i.e., false positives). ${ }^{3,4}$ It is the false positives that are known to increase both the cost and the duration of care. ${ }^{5}$ In addition, $22.4 \%$ of physicians tend to continue the empiric antibiotic therapy despite the availability of the culture data. ${ }^{6}$ Endimiani et $\mathrm{al}^{7}$ investigated this apparent aversion to the narrowing antibiotic coverage once culture data were noted. They found that only $67.9 \%$ of their study subjects (521 patients over a 2-year period) received the appropriate antibiotic treatment despite the availability of susceptibility results.

A large number of patients with febrile illness are evaluated in the ED. The indications for obtaining blood cultures from patients in the ED are even less clear. There are no impact data specific to the ED practice setting, and attempts to encourage informed clinical use of this test are few. It is invariably observed that blood cultures are more often ordered "because the patient has a fever" or "the admitting Registrar will want them" than for any expectation of clinical impact.

\section{MATERIALS AND METHODS}

This is a cross-sectional, record-based prospective study of admitted patients of Emergency department in a tertiary care teaching hospital. One ward from each medical (ward 22) and surgical (ward 1) emergency services was taken for the study. The data were collected for a period of 3 months from April 15 to July 15, 2012.

\section{Study Design}

The data were collected for a total period of 12 weeks in two phases, 6 weeks apart, in preintervention and postintervention respectively. All patients admitted in the above-said wards under study were followed up for a period of 3 months to find out: (1) Whether any blood cultures have been requisitioned, (2) whether he/ she was on antibiotics prior to requisitioned, (3) date of requisitioned blood culture, (4) date of receipt of reports, (5) response time, and (6) impact of culture reports on treatment.

An intervention was done personally by the researcher to ensure that all test reports reach the file of the patients within 6 hours of its generation. For this purpose, one hospital attendant round the clock was deputed to carry blood culture reports twice in his shift at predetermined interval from the Medical Microbiology Department to the respective files of the patients. Subsequently, the methodology as listed above was repeated for the patients in the study area for another 6 weeks (postintervention). Data were collected concerning patients' indication for culture, blood culture outcome, and change of treatment.

\section{Data Analysis Methods}

The data collected were analyzed to find out:

- No. of cultures that have been requisitioned before and after starting antibiotic therapy.

- No. of reports received (this included any test, received telephonically by the treating doctors, in addition to the report filled in the patient case sheet).

- No. of times treatment was started or changed in response to reports received.

- Percentage of utilization of blood culture was calculated based on the formula:

$\frac{\text { No. of cultures for which } \mathrm{Rx} \text { which was changed }}{\text { Total }} \times 100$

\section{Statistical Analysis}

This was conducted to find out the effect of the intervention on outcome in terms of the utilization coefficient specialty wise and age wise for all patients under study. Discrete data were calculated as $\mathrm{n}(\%)$ and continuous data (hospital stay) were presented as mean \pm standard deviation. Categorical data were compared using Pearson chi-square test or Fisher's exact test as appropriate.

All analyses were conducted using Statistical Package for the Social Sciences for Windows (version 15.0; SPSS Inc., Chicago, Illinois, USA). All statistical tests were twosided and performed at a significance level of $\alpha=0.05$.

\section{RESULTS}

A total of 104 blood cultures were sent for 71 patients over a period of 6 weeks in the preintervention phase. Out of the total 104 blood culture reports, 67 reports were sent before starting the antibiotics of the patients, and 37 blood culture reports were sent after the starting of antibiotics. Totally 131 blood culture reports were received after the process from the Microbiology Department. Out of total 131 reports received, no action was taken against 119 reports, 4 reports were received contaminated, and the change in treatment was done only in 8 reports.

In the postintervention phase, 70 patients were followed up over the period of 6 weeks. Totally 102 blood culture reports were sent in the Department of Microbiology. Out of 102 blood cultures, 61 blood samples were sent before the starting of the antibiotics and the 40 samples after the antibiotics. Totally 156 blood culture reports were received after the process from the Microbiology Department. Out of total 156 reports received, no action was taken against 140 reports, 6 reports received were contaminated and the change in treatment was done only in 10 reports.

Table 1 shows that out of total 71 patients admitted in the emergency wards, $36.6 \%(n=26)$ patients were already 
Table 1: Patients who were on antibiotics before coming to hospital

\begin{tabular}{llllll}
\hline & \multicolumn{2}{c}{ Preintervention } & & \multicolumn{2}{c}{ Postintervention } \\
\cline { 2 - 3 } & No. & $\%$ & & No. & $\%$ \\
\hline Yes & 26 & 36.6 & & 27 & 38.6 \\
No & 45 & 63.4 & & 43 & 61.4 \\
\hline Total & 71 & 100 & 70 & 100 \\
\hline
\end{tabular}

Table 3: Change in treatment of patients in response to blood culture report received

\begin{tabular}{llllll}
\hline & \multicolumn{2}{l}{ Preintervention } & & \multicolumn{2}{c}{ Postintervention } \\
\cline { 2 - 3 } & No. & $\%$ & & No. & $\%$ \\
\hline Yes & 7 & 9.9 & & 15 & 21.5 \\
No & 64 & 90.1 & & 55 & 78.6 \\
\hline Total & 71 & 100 & & 70 & 100 \\
\hline
\end{tabular}

on some form of antibiotics in the preintervention stage. In postintervention, this number was $28.6 \%(n=27)$.

Table 2 shows that out of a total of 131 blood culture reports received in the preintervention stage, $26.6 \%$ were positive, $70.2 \%$ were sterile, and $3.1 \%$ were contaminated. In postintervention, $14.1 \%$ were positive, $82.2 \%$ were sterile, and $3.8 \%$ reports were contaminated.

Table 3 shows that out of total 71 patients enrolled in the preintervention stage, the change in treatment after receiving blood culture was done in 7 (9.9\%) patients. This number increased to $15(21.4 \%)$ in case of postintervention.

Table 4 shows that out of total 104 blood culture samples that were sent in preintervention, 35 (33.3\%) blood culture reports returned positive and the change of treatment was done only in $8(7.6 \%)$ of culture reports. Similarly, in postintervention, total of 101 blood culture samples were sent, and 22 returned positive and change of treatment was done in $10(9.9 \%)$ reports. There
Table 2: Result of blood culture reports

\begin{tabular}{|c|c|c|c|c|}
\hline & \multicolumn{2}{|c|}{ Preintervention } & \multicolumn{2}{|c|}{ Postintervention } \\
\hline & No. & $\%$ & No. & $\%$ \\
\hline Positive & 35 & 26.7 & 22 & 14.1 \\
\hline Sterile & 92 & 70.2 & 128 & 82.1 \\
\hline Contaminated & 4 & 3.10 & 6 & 3.80 \\
\hline Total & 131 & 100 & 156 & 100 \\
\hline
\end{tabular}

was a marginal increase in change of treatment after intervention.

Table 5 shows that the utilization coefficient of blood culture was $12.1 \%$ in preintervention as compared with postintervention where it was $12.5 \%$. There was a marginal increase in utilization in surgery after the intervention. The utilization coefficient increased from 5.5 to $9.41 \%$ after intervention. The utilization coefficient in medicine was significant $(\mathrm{p}<0.05)$ and well utilized as compared with surgery.

Table 6 shows that in surgical patients, of the age group 16 to 60 , the utilization coefficient increased from 12 to $14.5 \%$ after intervention. In the age group of $>60$ years, the utilization coefficient decreased marginally from 16.7 to $14 \%$. In medicine, the utilization coefficient in both age groups increased after intervention, especially in the age group of $<60$ years, this number was $8.70 \%$.

\section{DISCUSSION}

Blood cultures are a commonly ordered test in ED. In recent years, a small number of papers have been published looking at the clinical utility of blood cultures as a test. These, however, are difficult to extrapolate to the ED setting, as they have either focused on hospital inpatients, patients in intensive care settings, or have "worked backward" from discharge diagnosis.

Table 4: Change in treatment according to the specific blood culture reports

\begin{tabular}{|c|c|c|c|c|c|c|}
\hline & \multicolumn{3}{|c|}{ Preintervention } & \multicolumn{3}{|c|}{ Postintervention } \\
\hline & Yes & No & & Yes & No & \\
\hline & No. (\%) & No. (\%) & Total & No. (\%) & No. (\%) & Total \\
\hline Positive & $8(7.6)$ & $27(26)$ & 35 (33.3) & $10(9.9)$ & $12(11.8)$ & $22(21)$ \\
\hline Sterile & $2(1.9 \%)$ & $63(60.5)$ & 65 & $14(13.8)$ & $59(57.1)$ & 73 \\
\hline Contaminated & 0 & $4(3.9)$ & 4 & 0 & $6(5.8)$ & 6 \\
\hline Total & 10 & 94 & 104 & 24 & 77 & 101 \\
\hline
\end{tabular}

Table 5: Specialty-wise utilization coefficient of blood culture

\begin{tabular}{llll}
\hline Specialty & $\begin{array}{l}\text { Preintervention } \\
(\%)\end{array}$ & $\begin{array}{l}\text { Postintervention } \\
(\%)\end{array}$ & $p$-value \\
\hline Surgery & 12.1 & 12.5 & 1 \\
Medicine & 5.5 & 9.41 & $0.038(<0.05)$ \\
\hline
\end{tabular}

Table 6: Specialty and age-wise utilization coefficient of blood cultures

\begin{tabular}{lllll}
\hline \multicolumn{5}{l}{ Age } \\
Specialty & group & Preintervention & Postintervention & p-value \\
\hline Surgery & $16-60$ & 12.0 & 14.5 & 0.75 \\
& $>60$ & 16.7 & 14.0 & 0.70 \\
Medicine & $16-60$ & 3.40 & 8.70 & $0.24(<0.05)$ \\
& $>60$ & 13.3 & 16.7 & $0.30(<0.05)$ \\
\hline
\end{tabular}


Blood cultures are routinely sent by treating physician as other common investigations are sent. There are a number of reasons for this behavior. Habit is a major factor. Hospital-based training reinforces the almost Pavlovian response that if a patient has fever, a blood culture should be taken. Also, there is a perception among ED staff that blood cultures should be taken because the inpatient unit will require them. The underlying assumptions that the inpatient unit is always right and that the blood cultures will alter management have yet to be proved. Studies have shown that in the presence of known major bacterial infection, blood cultures are only positive in up to $20 \%$ of cases. In most of these studies, multiple blood cultures have been taken, so the sensitivity of an individual test is likely to be lower than this. The pathology of the disease and thus the likelihood of bacteremia must also be considered. An additional factor is the prevalent fallacy that if blood cultures are not performed initially, "the boat has been missed."

In the present study, whether patients had taken antibiotics before coming to ED was assessed. It was found that out of total 71 patients, $26(36.6 \%)$ were already on some form of antibiotic in preintervention and $27(38.6 \%)$ patients out of total 70 patients in postintervention stage. Totally 37 blood cultures were sent for 26 patients and 40 blood culture for 27 patients in pre- and postintervention respectively, in spite of patients being already on antibiotics. Totally 18 and $15 \%$ of blood culture turned positive in pre- and postintervention respectively, in postantibiotic culture. But surprisingly, the change in treatment was not significant. So, sending blood culture after taking antibiotics has hardly any relevance in current clinical practice in ED.

A similar study was done by the Christopher J Grace et $\mathrm{al}^{8}$ about usefulness of blood culture of those who were receiving antibiotic therapy. They did not find any organism in $60 \%$ of the patients. The blood culture reports were either negative or contaminated. In $40 \%$ of the patients, there were some pathogens identified. But one condition was that the cultures were obtained during the first 72 hours of antibiotic therapy. Patients were excluded from the study if they had taken antibiotics during the 72 hours prior to the admission. If we compare with our study, it will be very difficult to get patients' history about the course of antibiotics therapy. This might be the reason for relatively high positivity rates in postantibiotic cultures.

Another study conducted by Murty and Gyaneshwari, ${ }^{9}$ from the Department of Microbiology, SV Medical College, Tirupati, Andhra Pradesh, found that administration of empirical antibiotics was already initiated by the time of collection of sample for culture in $71(66.35 \%)$ of the cases. Of these, only $6(8.45 \%)$ had positive cultures.
In the present study, out of the total 104 blood cultures that were sent for 71 patients in preintervention, 35 blood cultures returned positive and in postintervention, it was 22 blood culture reports. Normally, positivity rate is around 10 to $15 \%$. In our study, the reason for higher positivity might be due to inclusion of both true positive and false positive and also due to inclusion of coagulase-negative species, which is normally considered as contaminated. Only $7.6 \%$ of blood cultures taken in ED impact on management of the patient. This number increased to about $10 \%$ in the postintervention stage. It shows that there was a slight impact on treatment by providing blood culture reports within 6 hours of generation from the Microbiology Department. However, it was not very much significant. One more interesting result came in our study; in $2(1.9 \%)$ sterile blood cultures in preintervention stage and 14 (13.8\%) in postintervention, the physician changed the treatment of the patient. This might be due to the clinical judgment of the clinician or due to the deterioration of the patients.

The results of this study show that blood cultures obtained in the ED rarely yield a result that impacts on patient management. We found that the patient's clinical condition had a far stronger influence on change of antibiotic therapy than the result of blood culture.

Dr Birnbaumer's Centres for Medicare, Seattle, in his study about community-acquired pneumonia population, ${ }^{10-13}$ found that even when blood cultures are positive, they rarely have effect on clinical management. In $58 \%$ of patients with positive cultures, the empirical antibiotic course is continued despite culture results indicating that management can be changed. For majority of cases in which antibiotic coverage is changed, it is by clinical response, regardless of culture result.

Corbo et $\mathrm{al}_{1}{ }^{14}$ in their research paper on limited usefulness of initial blood cultures in community-acquired pneumonia found that in total, of 355 study participants, the management of only 18 cases $(5 \%)$ was changed by blood culture results. There were 151 patients (43\%) who had their antibiotic management changed by clinical improvement, and 23 patients (6\%) who had their antibiotic management changed by clinical deterioration.

In surgery, the utilization coefficient of blood culture was 12.1 in preintervention as compared with postintervention where it was 12.50 . There was a marginal increase in utilization. The utilization coefficient of blood cultures for the Medicine Department increased significantly in the postintervention phase. Although utilization was more in the Surgery Department, there was no effect of intervention. In medicine, there was significant impact of intervention. 


\section{Limitations of the Study}

As the study was confined to only two wards of the $\mathrm{ED}$, generalization of opinion cannot be made about the other emergency wards and even normal inpatient wards. False positives were not excluded in the study, which, if done, would further increase the wastage due to test results.

\section{Future Study}

- Costing of blood culture reports through the unit cost method using the $\mathrm{ABC}$ technique.

- Physician and surgeon behavior toward utilization of blood culture reports.

- Study of correlation between blood culture positivity and average length of stay of patients.

\section{REFERENCES}

1. Beekman SE, Diekema DJ, Chapin KC, Doern GV. Effects of rapid detection of bloodstream infections on length of hospitalization and hospital charges. J Clin Microbiol 2003 Jul;41(7):3119-3125.

2. Xu J, Kochanek KD, Murphy SL, Tejada-Vera B. Deaths: final data for 2007. Natl Vital Stat Rep 2010 May;58(19):1-19.

3. Wilson ML. Clinically relevant, cost-effective clinical microbiology. Strategies to decrease unnecessary testing. Am J Clin Pathol 1997 Feb;107(2):154-167.

4. Zwang O, Albert RK. Analysis of strategies to improve cost effectiveness of blood cultures. J Hosp Med 2006 Sep;1(5):272-276.
5. Bates DW, Goldman L, Lee TH. Contaminant blood cultures and resource utilization. The true consequences of falsepositive results. JAMA 1991 Jan;265(3):365-369.

6. Trenholme GM, Kaplan RL, Karakusis PH, Stine T, Fuhrer J, Landau W, Levin S. Clinical impact of rapid identification and susceptibility testing of bacterial blood culture isolates. J Clin Microbiol 1989 Jun;27(6):1342-1345.

7. Endimiani A, Tamborini A, Luzzaro F, Lombardi G, Toniolo A. A two-year analysis of risk factors and outcome in patients with bloodstream infection. J Infect Dis 2003 Feb;56(1):1-7.

8. Grace CJ, Lieberman J, Pierce K, Littenberg B. Usefulness of blood culture for hospitalized patients who are receiving antibiotic therapy. Clin Infect Dis 2001 Jun;32(11):1651-1655.

9. Murty DS, Gyaneshwari M. Blood cultures in paediatric patients: a study of clinical impact. Indian J Med Microbiol 2007 Jul-Sep;25(3):220-224.

10. Birnbaumer DM. Blood cultures aren't useful for managing immune competent CAP inpatients. JWEM 2004.

11. Campbell SG, Marrie TJ, Anstey R, Dickinson G, AckroydStolarz S. The contribution of blood cultures to the clinical management of adult patients admitted to the hospital with community-acquired pneumonia: a prospective observational study. Chest 2003 Apr;123(4):1142-1150.

12. Waterer GW, Jennings SG, Wunderink RG. The impact of blood cultures on antibiotic therapy in pneumococcal pneumonia. Chest 1999 Nov;116(5):1278-1281.

13. Chalasani NP, Valdecanas MA, Gopal AK, McGowan JE Jr, Jurado RL. Clinical utility of blood cultures in adult patients with community-acquired pneumonia without defined underlying risks. Chest 1995 Oct;108(4):932-936.

14. Corbo J, Friedman B, Bijur P, Gallagher EJ. Limited usefulness of initial blood cultures in community acquired pneumonia. Emerg Med J 2004 Jul;21(4):446-448. 